\title{
Virale hämorrhagische Fieber
}

Hinrich Sudeck

21.1 Einleitung - 90

21.2 Dengue-Fieber - 91

21.3 Krim-Kongo-Virus - 94

21.4 Ebola- und Marburg-Virus - 96

21.5 Andere Erreger von viralen hämorrhagischen Fiebern - 98

21.5.1 Gelbfieber - 98

21.5.2 Lassa-Fieber - 99

21.5.3 Südamerikanische hämorrhagische Fieber - 99

Literatur - 99 


\subsection{Einleitung}

Diese Erkrankungen, obwohl bis zum Sommer 2014 nur höchst selten importiert, sind für die allgemeine Öffentlichkeit und den öffentlichen Gesundheitsschutz von großer Bedeutung. Ursache ist eine heterogene Gruppe von Viren, die teils ein tierisches Reservoir haben und teils von Arthropoden als Vektoren von Tier zu Mensch oder von Mensch zu Mensch übertragen werden können. Manche von ihnen kommen nur regional begrenzt oder in spezifischen Ausbruchsregionen, die manchmal auch überraschend neu entstehen, vor. Andere wie hämorrhagisches Krim-Kongo-Fieber, Dengue- und Chikungunya-Fieber breiten sich durch internationalen Reiseverkehr und Warenaustausch in den letzten Jahren rapide und kontinuierlich mit ihren Vektoren auch in gemäßigten Klimazonen aus.

Vier bedeutsame Virusfamilien stellen mit 7 Gattungen die wichtigsten Erreger (• Tab. 21.1). Von diesen Erkrankungen sind Ebola-, Marburg-, Lassa-, Krim-Kongo- und Machupo-Fieber von Mensch zu Mensch übertragbar.

Impfpräventabel ist bei uns nur das Gelbfieber, regional sind Impfstoffe für Krim-Kongo-Virus in Bulgarien, Juninund Machupo-Fieber in Argentinien und für Hantavirus in Ostasien verfügbar. Eine Dengue-Virus-Vakzine ist in klinischen Studien in Erprobung, bisher aber noch nicht sehr vielversprechend.

\section{- Pathogenese}

Die Pathogenese lässt sich - am Beispiel der Ebola-Infektion - vereinfacht und schematisch folgendermaßen beschreiben: Ein direkter Gewebeschaden entsteht nach der perkutanen Infektion durch Bindung des Virus an C-Typ-Lektine auf Leberzellen, Makrophagen und Lymphknotenoberflächen durch toxische Effekte viraler Glykoproteine und anderer Matrixproteine. Ein indirekter Schaden wird hervorgerufen durch:

- Unterdrückung von Abwehrmechanismen (= ausbleibende Produktion von Zytokinen) der Makrophagen und besonders der dendritischen Zellen

- Blockade der Aktivierung von T-Zellen durch dendritische Zellen

- Bystander-Apoptose

- Zytokin- und Chemokinfreisetzung durch infizierte Makrophagen mit Erhöhung des „tissue factor“, dies führt zu einer DIC („disseminated intravascular coagulation“, disseminierte intravasale Gerinnung)

Zusätzlich kommt es zu einem klinisch fatalen Capillary-leak-Syndrom durch Schädigung von Endothelzellen. Die Auslösung von Zytokinkaskaden und die Aktivierung des eigenen Immunsystems tragen zum schnellen Multiorganversagen bei, das in der Regel - eher als Verbluten - die Todesursache ist. Einzelne virale hämorrhagische

\begin{tabular}{|c|c|}
\hline \multicolumn{2}{|c|}{$\begin{array}{l}\text { Tab. } 21.1 \text {. Die wichtigsten viralen hämorrhagischen } \\
\text { Fiebererkrankungen }\end{array}$} \\
\hline Virusfamilie & Wichtigste Erkrankungen \\
\hline \multirow[t]{2}{*}{ Flaviviren } & Gelbfieber \\
\hline & Dengue-Fieber \\
\hline \multirow[t]{3}{*}{ Bunyaviren } & Rift-Tal-Fieber \\
\hline & Krim-Kongo-Fieber \\
\hline & Hantavirusinfektion \\
\hline \multirow[t]{4}{*}{ Arenaviren } & Lassa-Fieber \\
\hline & $\begin{array}{l}\text { Junin-Fieber (argentinisches hämorrhagi- } \\
\text { sches Fieber) }\end{array}$ \\
\hline & $\begin{array}{l}\text { Machupo-Fieber (bolivianisches hämorrha- } \\
\text { gisches Fieber) }\end{array}$ \\
\hline & $\begin{array}{l}\text { Guanarito-Fieber (venezolanisches hämo- } \\
\text { rrhagisches Fieber) }\end{array}$ \\
\hline \multirow[t]{2}{*}{ Filoviren } & Hämorrhagisches Ebola-Fieber \\
\hline & Hämorrhagisches Marburg-Fieber \\
\hline
\end{tabular}

Fieber haben einen eigenen charakteristischen Verlauf, allen gemeinsam ist aber der unspezifische, wenig dramatische Beginn der Symptomatik (selbst Fieber kann fehlen). Hautveränderungen, meist im Sinn eines flüchtigen feinen Exanthems, sind möglich.

$>$ Eine Hautbeteiligung ist bei den viralen hämorrhagischen Fiebern nicht obligat. Die Symptome können Blutungsstigmata von Haut und Schleimhaut sein oder unspezifische Exantheme.

Es besteht Meldepflicht nach $₫ 6$ (virale hämorrhagische Fieber) und $₫ 7$ IfSG.

Arbovirosen und virale hämorrhagische Fieber

- Die maximale Inkubationszeit für diese Erkrankungen liegt bei 21 Tagen.

- Die Anamnese sollte die genaue Reiseroute und Expositionsrisiken erfassen.

- Thrombozytopenie und Leberbeteiligung sind mögliche Alarmzeichen.

- Ein prompter Malariaausschluss ist obligat. Dengue-Schnelltests stehen zur Verfügung.

- Im Zweifel sollten Isoliermaßnahmen und Techniken des „barrier-nursing“ zum Einsatz kommen. Zudem sollte immer Rücksprache mit einem Kompetenzzentrum gehalten werden (Tropeninstitute unter - www.dtg.org und Homepage des STAKOB über www.rki.de). 


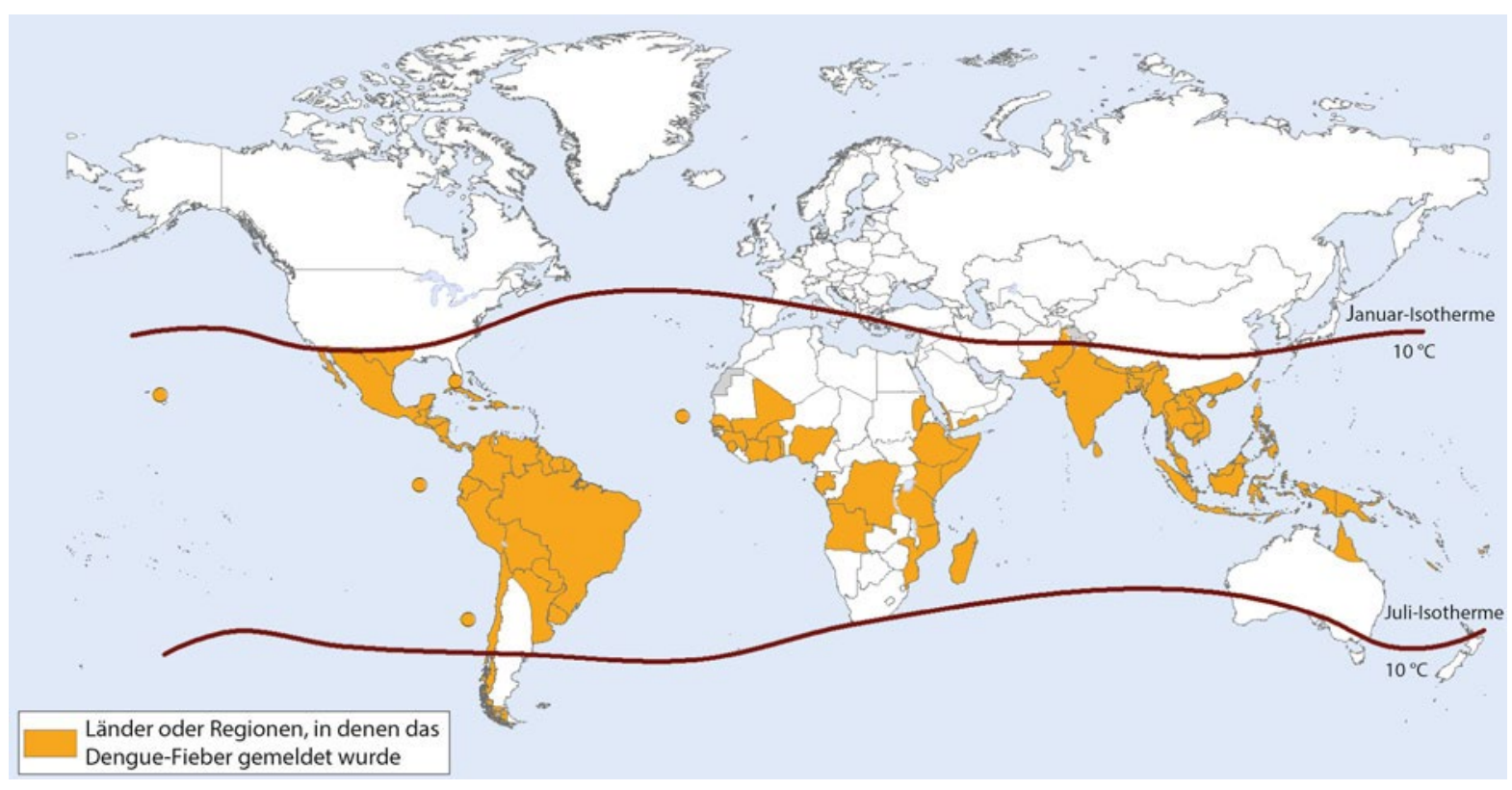

- Abb. 21.1 Verbreitungsgebiet des Dengue-Fiebers im Jahr 2013. (Adaptiert nach WHO 2014)

\subsection{Dengue-Fieber}

Dies ist eine der klassischen Arbovirusinfektionen (Arbo = ,arthropod born“, Arthropoden sind Gliederfüßler, infrage kommen vor allem Stechmücken und Zecken). Sie wird von weiblichen Mücken der Spezies Aedes aegypti und A. albopictus übertragen, die an einem virämischen Patienten das Virus aufgenommen haben. Eine Umbenennung der Mücken führte auch zur Bezeichnung Stegomyia. Beide Arten sind sowohl in Gebäuden als auch in der Natur auf der Suche nach potenziellen Opfern und sind tagsüber als auch abends aktiv. Sie saugen das Blut jeweils nur kurz, dafür aber an vielen Menschen. Schon kleine wassergefüllte Behälter reichen als Brutplatz aus. Ein einmal infizierter Moskito bleibt lebenslang infektiös.

Eine Übertragung durch Bluttransfusion ist möglich, Infektionen im Rahmen von Transplantationen oder transplazentar, intrapartal oder mukokutan sind sehr selten.

\section{- Häufigkeit und Vorkommen}

Mit 4 Subtypen (inzwischen gibt es Meldungen über einen fünften) ist Dengue weltweit in den Tropen und Subtropen verbreitet (•Abb. 21.1). Ein Viertel der Weltbevölkerung hat ein Erkrankungsrisiko, und die WHO geht von knapp 100 Millionen Fällen pro Jahr aus. Wahrscheinlich liegt die Dunkelziffer bei weiteren 200 Millionen Fällen. Die Subtypen 2 und 3 gelten als die Erreger der schwerwiegendsten Verläufe.

Dengue-Antikörper sind auch in Primaten in Afrika und Asien gefunden worden, wobei die Tiere nicht erkranken. Mit dem Auftauchen von Aedes (Stegomyia) albopictus als Vektor ist die Ausbreitung auch in gemäßigtere Zonen möglich geworden, was durch Fälle in Südfrankreich und Kroatien belegt wird.

Insgesamt ist das Dengue-Fieber die sich am schnellsten ausbreitende Arbovirusinfektion in der Welt; die Fallzahl in Deutschland hat mit über 600 Fällen im Zeitraum 2013/14 die Zahl der Malariafälle überholt. Typische Infektionsgebiete sind Asien (Indien, Thailand, Indonesien) und Südamerika (Brasilien, Karibik) - auch Großstädte wie Bangkok und Singapur sind betroffen. 2013 kam es zu Fällen in China und Florida, 2014 zu einem Anstieg der Fallzahlen vor allem in Malaysia und auf den pazifischen Inseln Fiji, Vanuatu und Cook Islands. Für die sichere Abwicklung der Fußballweltmeisterschaft in Brasilien galt Dengue-Fieber als bedrohlicher Risikofaktor.

\section{- Klinik}

Die klinischen Erscheinungen reichen von der asymptomatischen, aber virämischen Infektion bis zu schweren tödlichen Verläufen mit Hämorrhagien und Multiorganversagen, die Erkrankung wird dann als hämorrhagisches Dengue-Fieber (DHF) oder auch Dengue-Schocksyndrom (DSS) bezeichnet. Gemäß WHO werden die Schweregrade in 3 Stufen unterteilt:

- Vermutliches/laborbestätigtes Dengue-Fieber

- Dengue-Fieber mit Alarmzeichen

- Dengue-Fieber mit „plasma leakage, severe bleeding, severe organ involvement"

Nach einer Inkubationszeit von 2-7 Tagen (bis 2 Wochen) kommt es abrupt zu verschiedenen, anfangs unspezifi- 

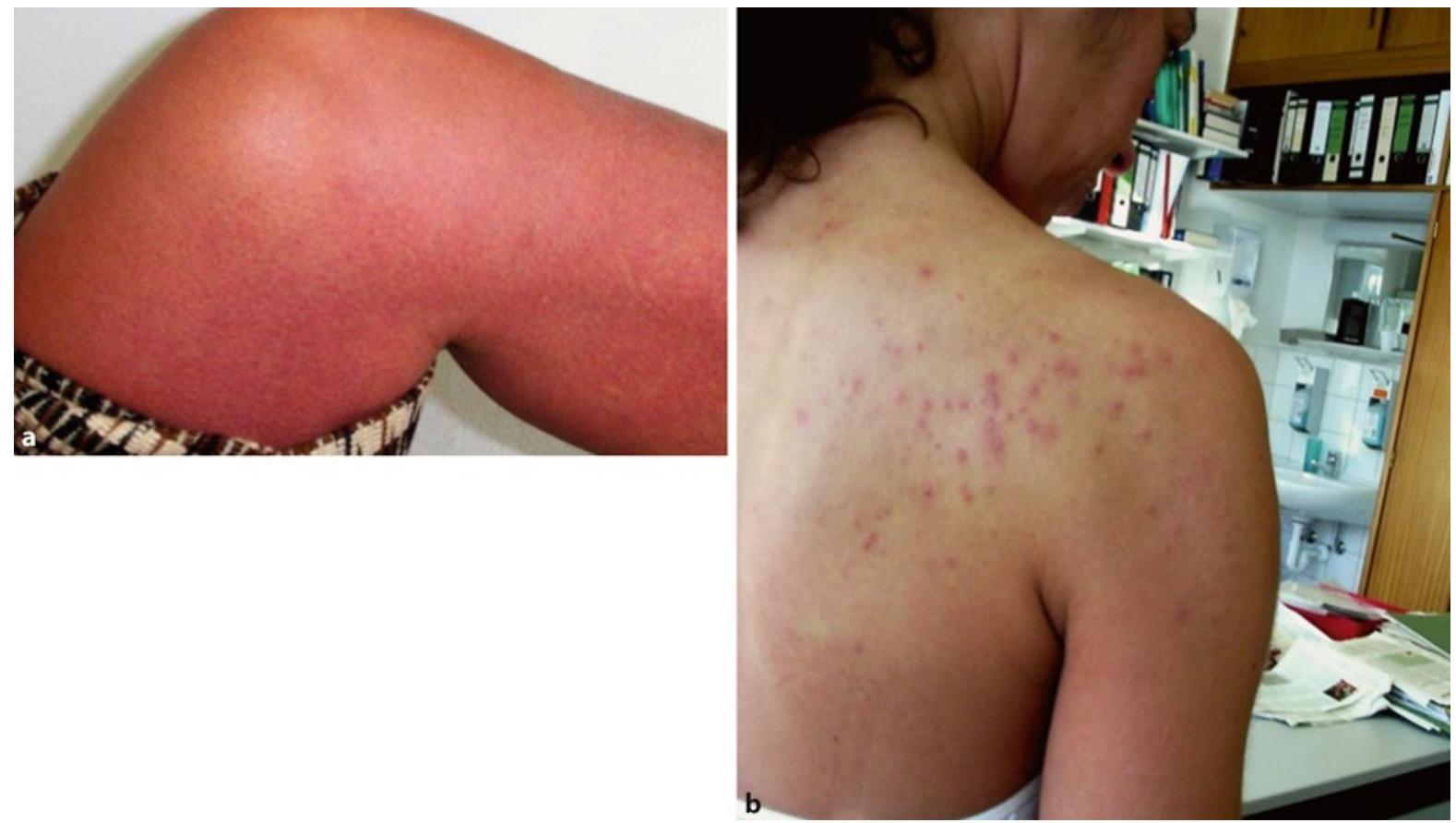

- Abb. 21.2a,b Hautbefunde bei Dengue-Fieber. a Initial exanthematös, b später makulopapulös

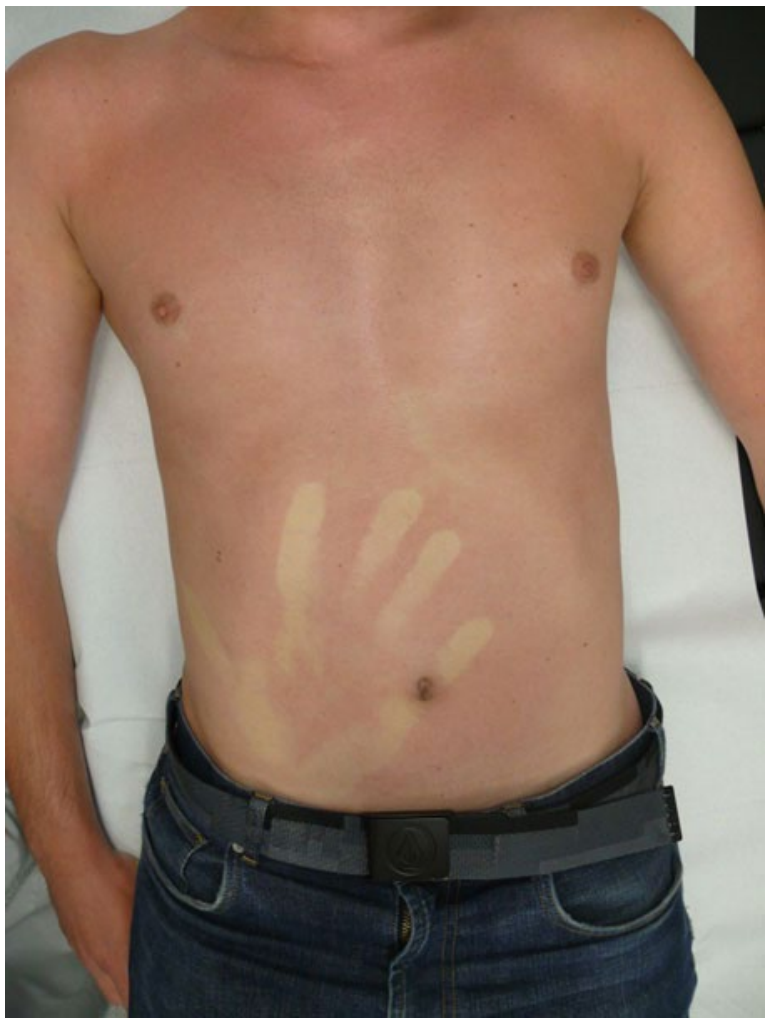

- Abb. 21.3 Negativer Dermografismus schen, fieberhaften Allgemeinsymptomen. Kinder haben initial meist nur ein unspezifisches Fieber, bei Erwachsenen ist die Erkrankung zusätzlich gekennzeichnet durch Auftreten von retroorbitalen Kopfschmerzen, Gliederschmerzen, (schwerem) allgemeinem Krankheitsgefühl und auch von Schüttelfrösten. Ein zweigipfliger Fieberverlauf („saddle back fever“) mit kurzzeitiger Entfieberung nach dem zweiten Tag ist nicht selten, aber nicht obligat. Es besteht eher eine Bradykardie als eine fiebertypische Tachykardie.

Pathognomonisch kann bei entsprechender Expositionsanamnese die Hautsymptomatik wie folgt sein: Neben einer kutanen Hypersensitivität und flächigen Rötung, die von den Patienten gelegentlich auch als Symptom eines Sonnenbrands eingeschätzt wird, kommt es zum Auftreten eines anfänglich feinfleckigen, flächigen, oft nicht sehr eindrucksvollen Exanthems (• Abb. 21.2a), das an Tag 5-6 morbilliform bis makulopapulös ( Abb. 21.2b) werden kann und nicht juckt. Handflächen und Fußsohlen sind ausgespart. Typisch ist auch ein negativer Dermographismus (• Abb. 21.3).

Bei den schweren Verlaufsformen DHF/DSS, die in der Regel bei der Zweitinfektion mit einem anderen Virustyp oder bei Vorhandensein maternaler Antikörper auftreten („antibody dependend enhancement of infection"), kommt es an Tag 2-5 zu einer akuten Verschlechterung mit Ödemen und Ergüssen durch Flüssigkeitsverschiebungen in den Extravasalraum, messbar anhand eines starken Anstiegs des Hämatokrits bei gleichzeitig dramatisch fal- 
lenden Thrombozyten. Ferner kommt es zu Petechien, Schleimhautblutungen (• Abb. 21.4), metabolischer Azidose und Multiorganversagen, in erster Linie mit Leberbeteiligung und ZNS-Komplikationen. Positive Prädiktoren für einen solchen Verlauf, der unbehandelt in $10 \%$ und behandelt in 1-2\% der Fälle letal ist, waren bei thailändischen Kindern:

- positiver Tourniquet-Test (auch als Rumpel-Leede-Phänomen bezeichnet)

- druckschmerzhafte Hepatosplenomegalie

- konjunktivale Injektion/Flush

Die Laborwerte waren jedoch nicht prädiktiv. Eine ausführliche Metaanalyse zu diesem Thema veröffentlichten Huy et al. (2013). Anhaltspunkte für ein erhöhtes Infektionsrisiko und einen schwereren Verlauf in der Schwangerschaft lieferten Machado et al. (2013).

\section{- Diagnostik}

Der molekulargenetische Nachweis von RNA oder dem Dengue-NS-1-Antigen ist schon vor dem Beginn der Symptome und bis etwa Tag 4 der Erkrankung möglich. IgG- und IgM-Antikörper sollten ab Ende der ersten Krankheitswoche bestimmt und im Verlauf wiederholt werden, wenn das erste Ergebnis nicht schon zur sicheren Diagnosestellung geführt hat. Kreuzreaktionen mit anderen Flaviviren sind zu beachten, niedrige Anfangstiter sind oft durch eine vorangegangene Gelbfieberimpfung bedingt.

Bei der Zweitinfektion kann die Ausbildung von IgM-Antikörpern fehlen oder nur gering sein. Kommerziell verfügbare Schnelltests stehen zur Verfügung. Sie weisen ausreichende Sensitivität und Spezifität auf und sind in der akuten Aufnahmesituation hilfreich, besonders bei positivem Ergebnis.

Im klinisch-chemischen Labor findet sich häufig eine Erhöhung der Transaminasen und der LDH. Im Blutbild ist eine Thrombozytopenie durch Infektion von Progenitorzellen und vermehrten Abbau der Thrombozyten sowie eine Lymphopenie zwar charakteristisch für einen viralen Infekt, aber nicht für einen spezifischen Erreger, sodass das differenzialdiagnostische Spektrum relativ weit sein kann.

Die sorgfältige Reise-und Expositionsanamnese kann wegweisend sein. Im Zweifelsfall sollten die Patienten bis zum Eingang der Befunde isoliert und Maßnahmen des „barrier-nursing“ und Nutzung von „personal protective equipment" ergriffen werden.

\section{- Differenzialdiagnose}

Wichtige Differenzialdiagnosen sind Malaria, Typhus, gramnegative Sepsis, Leptospirose, Infektion mit anderen Arboviren wie Zika-Virus und Sindbis-Virus und darüber hinaus auch hochinfektiöse, gefährliche virale hämorrhagische Fieber wie Ebola- (Afrika), Krim-

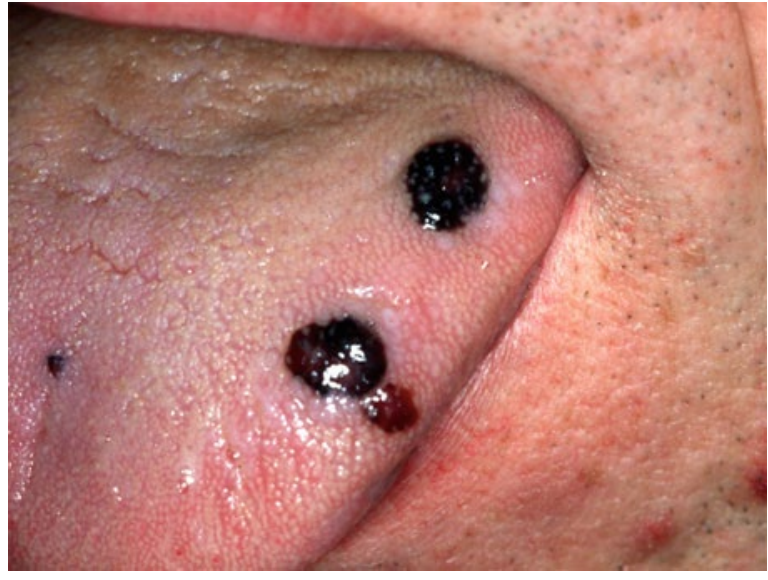

- Abb. 21.4 Typische Schleimhautblutungen auf der Zunge bei absoluter Thrombozytopenie

Kongo- (Balkan, naher Osten, Asien, Afrika) oder Lassa-Virus-Infektionen (Afrika). Bei Rückkehrern aus Südamerika kommen regional vorkommende seltene Viruserkrankungen infrage. Bei Südostasienreisenden sind Infektionen mit Orientia tsutsugamushi eine wichtige Differenzialdiagnose, gerade auch wegen des Exanthems. Prompter Malariaausschluss ist immer wichtig; eine Thrombozytopenie kann auch Ausdruck einer Malaria sein. Koinfektionen mit Chikungunya-Virus oder Malaria kommen vor.

(2) Symptome, die erst 2 Wochen oder länger nach Verlassen des Endemiegebiets auftreten, sind in der Regel kein Dengue-Fieber.

\section{- Therapie}

Die Therapie ist rein supportiv-symptomatisch, kann aber gerade bei den schweren Verlaufsformen lebensrettend sein. Als Infusionslösungen für den Flüssigkeitsersatz kommen kolloidale und kristalloide Lösungen infrage. Kommt es zu Blutungen, müssen Thrombozyten, Blut und Blutprodukte eingesetzt werden, was in nicht industrialisierten Ländern immer mit dem Risiko weiterer Infektionen einhergehen kann (• Abb. 21.5). Sehr detaillierte und stadienadaptierte Hinweise zur Therapie gibt das im Internet abrufbare WHO-Manual ( $\triangleright$ http:// whqlibdoc.who.int/publications/2009/9789241547871_eng. pdf?ua=1).

Der Nutzen einer Steroidgabe konnte in einer Cochrane-Analyse aus dem Jahr 2014 nicht belegt werden (Zang und Kramer 2014). Reisende in die Endemiegebiete sollten daher immer besonders eindringlich auf die Bedeutung des Mückenschutzes aufmerksam gemacht werden, insbesondere wenn es schon in der Vergangenheit zu einer Erstinfektion gekommen ist. In diesen Fällen muss aber nicht von weiteren Reisen abgeraten werden. 


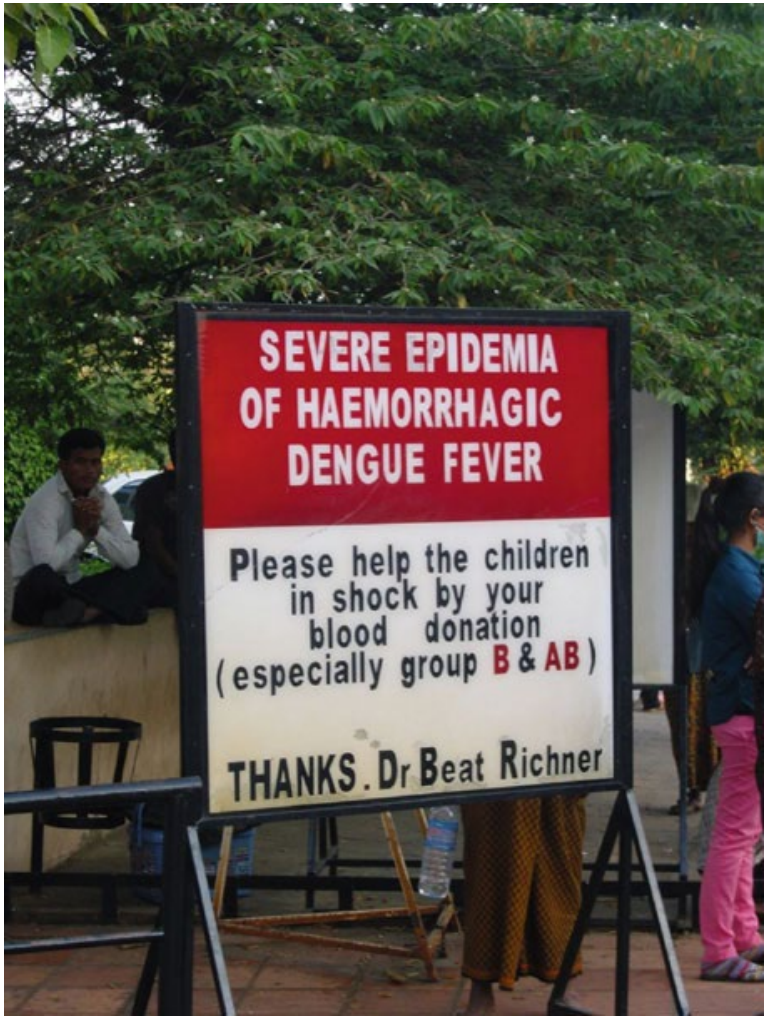

- Abb. 21.5 Aufruf zur Blutspende vor dem Kinderkrankenhaus in Siem Reap/Kambodscha

\subsection{Krim-Kongo-Virus}

Das hämorrhagische Krim-Kongo-Fieber („Crim-Congo hemorrhagic fever", CCHF) ist eine der klassischen Arbovirosen („tick-born“) und nach dem Dengue-Fieber die am weitesten verbreitete (• Abb. 21.6). Das Krim-Kongo-Virus (CCV) ist auch eines der historisch am frühesten beschriebenen hämorrhagischen Fieberviren (HFV). Es existieren mehrere Subtypen.

\section{- Pathogenese}

Ein besonderes Charakteristikum dieser an sich zoonotischen Erkrankung ist ihr Übertragungsmodus: Einerseits kann das Virus bei Blutkontakten und Sekretkontaminationen von Mensch zu Mensch, insbesondere auch nosokomial, übertragen werden, aber auch durch Zeckenstiche, vorwiegend durch Hyalomma-Zecken (• Abb. 21.7). Das natürliche Reservoir des Krim-Kongo-Virus sind Paarhufer, Nagetiere, Hasenartige und Vögel. Es gehört wie das Hantavirus zur Gruppe der Bunyaviren.

Die Pathogenese des CCHF wird oft mit der von Ebola verglichen. Wie alle hämorrhagischen Fieberviren wirkt auch das Krim-Kongo-Virus auf 2 Ebenen: Direkte Zellschädigung in den Organen durch virusbedingte zytopathische Effekte mit Endothelschädigung geht einher mit einer Aktivierung des Immunsystems mit Freisetzung von Zytokinen, Chemokinen, NO und anderen Mediatoren und resultiert in einem programmierten Zelltod, einer Aktivierung der Gerinnungskaskade mit Entwicklung einer disseminierten intravasalen Gerinnung (DIC) und Multiorganversagen.

\section{- Häufigkeit und Vorkommen}

Seit der Erstbeschreibung 1967 sind etwa 140 Ausbrüche mit mehr als 5000 betroffenen Patienten in 52 Ländern (Asien, Afrika, Mittlerer Osten, Südosteuropa) beschrieben worden. Dabei sind die Ausbrüche in der Türkei und die starke saisonale Zunahme der Fälle zwischen April und Juli besonders im Kosovo 2008, 2009 und 2010 bemerkenswert. Nosokomiale Übertragungen auf Ärzte und Pflegepersonal haben in den letzten Jahren besonders bei Ausbrüchen in der Türkei, in Mauretanien, Bulgarien, Indien und Pakistan stattgefunden, teils auch mit Todesfolge.

In der Region Pakistan, Afghanistan und Indien nimmt die Zahl der Erkrankungsmeldungen stark zu, aber auch in Spanien hat es im Jahr 2010 einen ersten Nachweis von $\mathrm{CCV}$ in Hyalomma-Zecken gegeben (Estrada-Peña et al. 2012). Im Südsudan wurde 2010 nach vielen Verdachtsfällen zum ersten Mal der Nachweis einer nosokomialen Übertragung nach Erkrankung eines Schlachters molekulargenetisch gesichert (Elata et al. 2011). Nach Zeckenstich im Rahmen eines Einsatzes im Süden Afghanistans verstarb 2009 ein amerikanischer Soldat.

\section{- Klinik}

Das klinische Bild ähnelt dem anderer Infektionen mit hämorrhagischen Fieberviren wie Ebola-, Marburg- oder Lassa-Viren. Die Ausprägung hängt von der Infektionsdosis (minimal 1-10 Viren) und dem Infektionsweg ab, ganz sicher auch vom Virustyp. Nach einer Inkubationszeit von etwa 3-7 Tagen, maximal dokumentiert sind 13 Tage, kommt es in der prähämorrhagischen Phase zu Kopfschmerzen, Fieber ohne Rhythmik, Myalgie und Schwindel. Diarrhö, Übelkeit und Erbrechen können hinzukommen, eine Hyperämie der Haut, unspezifische Exantheme und Konjunktivitis sind beschrieben. Nach etwa 3 Tagen läuft die Erkrankung dann entweder unspektakulär aus oder geht in die hämorrhagische Phase über, die abrupt beginnt und mit Blutungen in allen Organen einhergeht ( $\bullet$ Abb. 21.8); teils sind auch innere Blutungen ohne äußere Stigmata beschrieben.

Wird diese Phase überlebt, so kommt es etwa 1020 Tage nach Erkrankungsbeginn zur Rekonvaleszenzphase, die mit Allgemeinsymptomen einhergeht. Von Fällen aus dem Kosovo aus dem Ausbruch 2009/2008 wurde im Nachhinein von einer erheblich prolongierten Virämie bis zum Tag 36 nach Erkrankungsbeginn berichtet. In dieser Hinsicht ähnelt das Krim-Kongo-Virus anderen hämorragischen Fieberviren wie Lassa- oder Marburg-Vi- 


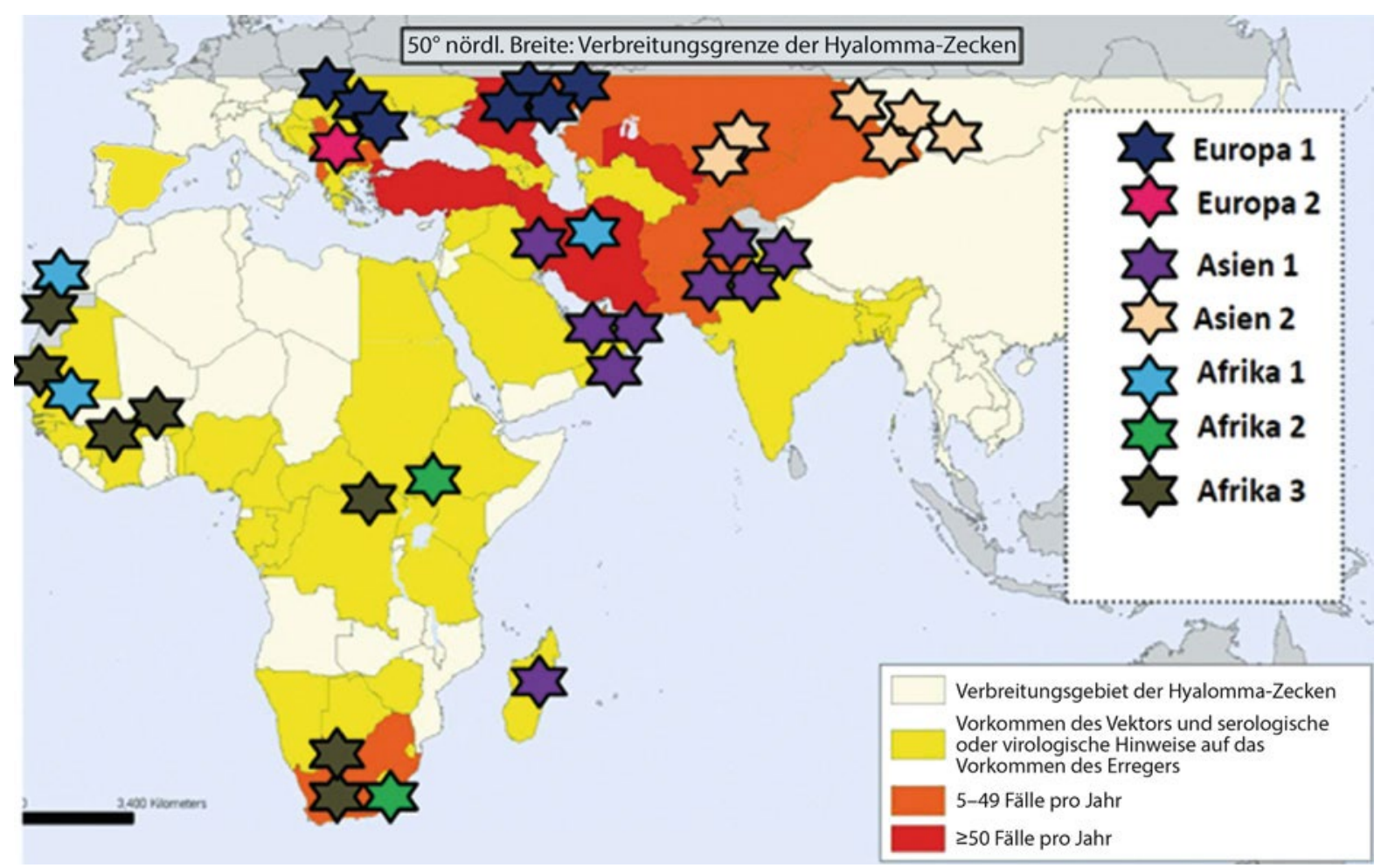

- Abb. 21.6 Verbreitungsgebiet des Krim-Kongo-Virus und seiner Subtypen (Sterne). (Adaptiert nach WHO 2008)

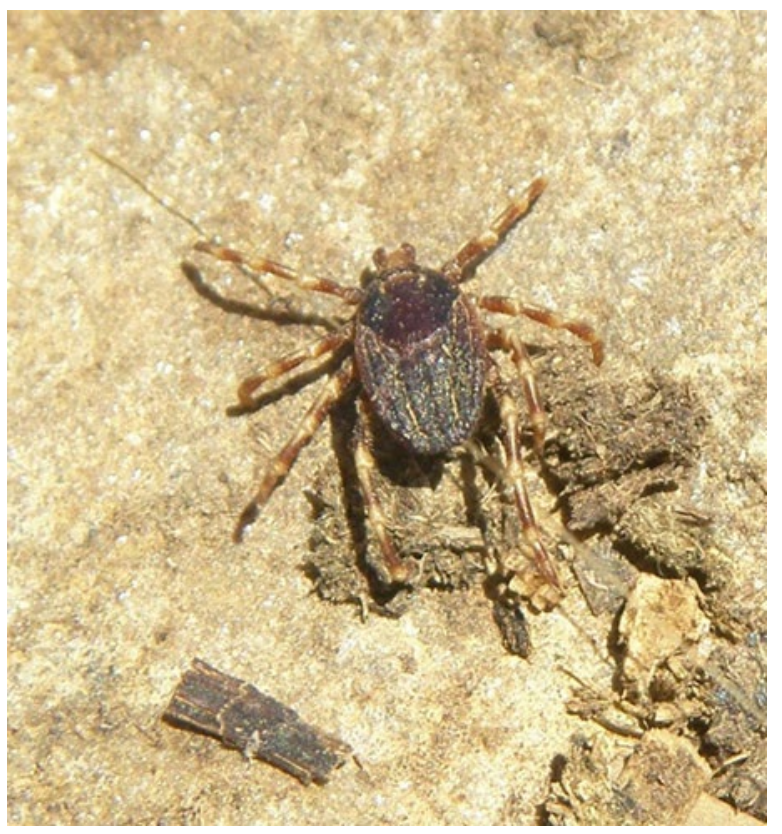

- Abb. 21.7 Hyalomma marginatum, der wichtigste Vektor für das Krim-Kongo-Virus. (๑ PD Dr. A. Krüger, Hamburg)

rus. Türkische Kollegen haben versucht, eine Einschätzung der Prognose anhand einiger einfacher Laborparameter zu ermöglichen (Dokuzoguz et al. 2013). Als ungünstige Prognoseparameter gelten:

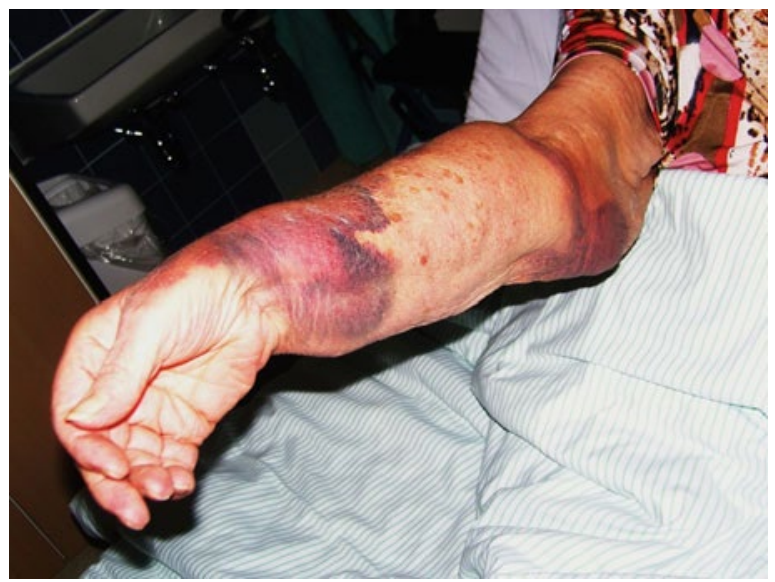

- Abb. 21.8 Typische Blutungsstigmata bei einer Infektion mit dem Krim-Kongo-Virus. (๑ Hinrich Sudeck, Hamburg)

- Thrombozyten $<20.000 / \mathrm{mm}^{3}$

- Alaninaminotransferase (ALT) $>900 \mathrm{U} / \mathrm{ml}$ oder Aspartataminotransferase (AST) $>700 \mathrm{U} / 1$

- aktivierte partielle Thromboplastinzeit $($ aPTT) $>60 \mathrm{~s}$ oder Fibrinogen $<110 \mathrm{mg} / \mathrm{dl}$

Von einigen Autoren wurden noch gastrointestinale Blutung, Somnolenz und Splenomegalie hinzugefügt und Patienten, die an CCHF starben, hatten signifikant höhere IL-6 und TNF- $\alpha$-Spiegel als Überlebende. 
Die Letalität ist regional unterschiedlich und wird meist mit 20-50\% angegeben (WHO: 30\%). Der Tod tritt in der Regel in den ersten 2 Woche ein. Im Falle eines afghanischen, in London versorgten Patienten war die Krankheitsdauer mit 5 Tagen sehr kurz (Bond 2012).

\section{- Diagnostik}

Die Anamnese des Patienten, der unter Umständen im weiteren Verlauf schnell nicht mehr auskunftsfähig sein wird, muss „ziseliert und mit Liebe“ erfolgen, unter Erfassung aller Expositionsrisiken. Neben Zeckenkontakten muss nach Kontakt zu Erkrankten, Tieren und nach Outdoor-Aktivitäten gefragt werden. Kontakt zu Einheimischen und Tieren ist ein wichtiger Expositionsfaktor. Selbst in wüstenhaften Regionen gibt es Ziegen- oder Schafhaltung, damit sind auch Zeckenvorkommen möglich, womit eine Exposition gegeben ist.

( Die Labordiagnostik muss unter Beachtung der entsprechenden Biosafety-Regelungen erfolgen.

Ein Nachweis von IgM- und IgG-Antikörpern mittels ELISA ist routinemäßig etabliert und wird am sechsten bis siebten Tag positiv. Die IgM-Antikörper persistieren über 4 Monate, IgG ist bis zu 5 Jahre nach Infektion noch nachweisbar. Schnell verstorbene Patienten zeigen keine messbare Immunantwort.

Um eine nosokomiale Ausbreitung zu verhüten, ist eine möglichst schnelle Diagnosestellung essenziell. Sie erfolgt heute in allen Zentren innerhalb von $8 \mathrm{~h}$ mittels PCR, meist als Multiplex-PCR. Virusisolation und Anzucht sind möglich, müssen aber unter BSL-4-Bedingungen erfolgen und dauern 2-5 Tage (BSL 4 = Bio-Safety-Level 4, Hochsicherheitsstufe für Labore oder klinische Einrichtungen).

\section{- Differenzialdiagnose}

Wichtige Differenzialdiagnosen sind alle anderen viralen hämorrhagischen Fieber, soweit sie in der entsprechenden Region vorkommen. Wichtige bakterielle Erreger sind vor allem Rickettsien (muriner Typhus und Fleckfieber sowie Infektionen durch Orientia tsutsugamushi in Asien), Leptospiren, Borrelia recurrentis, Meningokokken sowie Blutparasitosen wie Malaria und akute Trypanosomiasis (Zentralafrika).

\section{- Therapie}

Bei der Versorgung dieser Patienten müssen soweit wie möglich die Richtlinien des „barrier nursing“ zur Anwendung kommen - Eigenschutz hat Vorrang (• Abb. 21.9). Schon bei Verdacht (Meldepflicht!) ist daher zu einem tropenmedizinischen oder virologischen Zentrum mit Expertise für diese Erkrankungen Kontakt aufzunehmen $(\triangleright$ www.stakob.de, $\triangleright$ www.rki.de). Bis zur Verlegung mit-

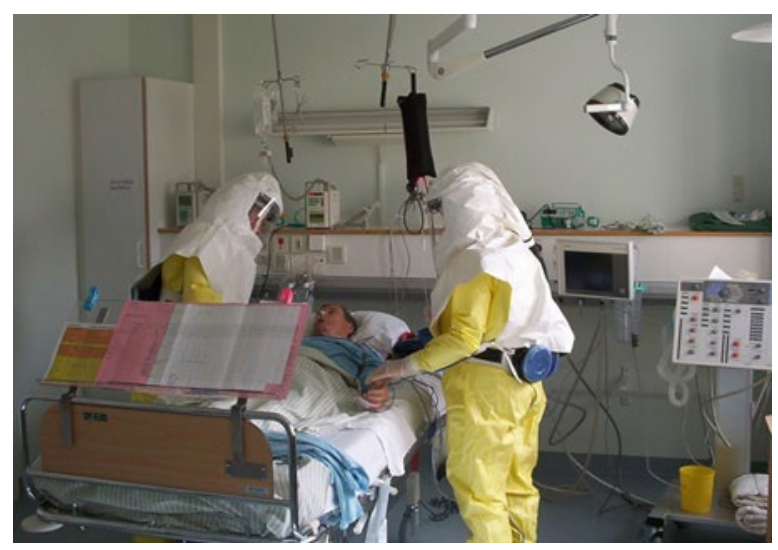

- Abb. 21.9 „Barrier nursing“: Arbeiten in der persönlichen Schutzausrüstung

tels eines Spezialtransportes muss der Patient strikt isoliert werden. Das zuständige Gesundheitsamt sollte von vornherein miteinbezogen werden.

Neben einer aggressiven supportiven Behandlung von Blutungen und Multiorganversagen kommt Ribavirin, ein 1972 synthetisiertes Purin-Nukleosid-Analogon, zur Anwendung. Als Polymerasehemmer verhindert es die Vermehrung von RNA- und DNA-Viren in vitro, seine Effizienz in vivo ist aber bis heute umstritten und mag auch vom möglichst frühen Zeitpunkt der Anwendung abhängen. Eine Fallvergleichsstudie aus der Türkei (Duygu et al. 2012) hat bei retrospektiver Analyse keinen Überlebensvorteil für orales Ribavirin erbracht. Ein international akzeptiertes Dosierungsschema wurde von der WHO sowohl für die orale als auch für die teure intravenöse Anwendung empfohlen.

Weitere angewandte Therapieoptionen wie die Gabe von Immunsera oder Subunit-spezifischen monoklonalen Antikörpern sind genauso wenig durch kontrollierte Studien belegt bzw. nicht belegbar, da ethische Grundsätze einer plazebokontrollierten Studie auf Dauer entgegenstehen.

\section{- Prophylaxe}

Prävention ist möglich durch strengen Zeckenexpositionsschutz, besonders in den bekannten Endemiegebieten. In Bulgarien steht eine Impfung zur Verfügung. Die postexpositionelle Einnahme von Ribavirin ist möglich.

\subsection{Ebola- und Marburg-Virus}

- Häufigkeit und Vorkommen

Beide Viren, Ebola- und Marburg-Virus, gehören zu den Filoviren und kommen nur in Afrika vor (eine nicht humanpathogene Spezies der 5 bekannten Ebola-Spezies allerdings auch bei Affen in Südostasien). Das Marburg-Virus trat zum ersten Mal 1967 in Marburg in Erscheinung, 

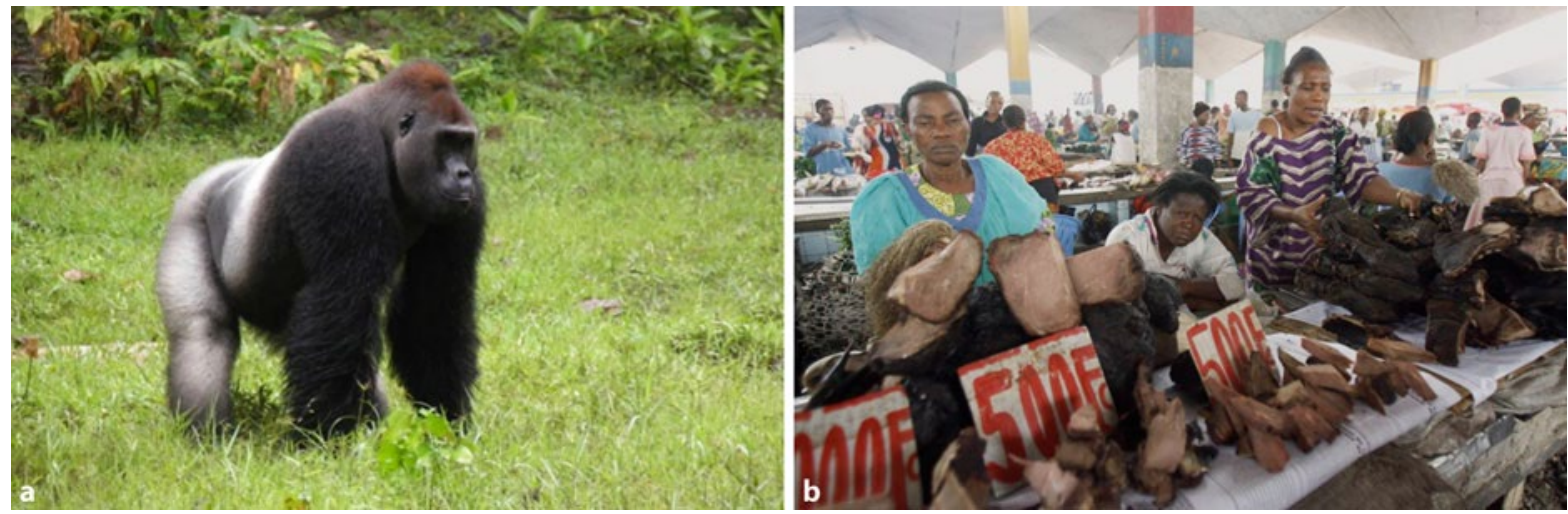

- Abb. 21.10a,b Quellen für die Infektion mit Ebola-Viren. a Gorilla in Zentralafrika (๑ Flottillenarzt Dr. A. Fritsch), b Bushmeat-Stand im Kongo (๑ Fachbereich Tropenmedizin der Bundeswehr)

nachdem importierte infizierte Affen aus Uganda das Personal einer Forschungseinrichtung angesteckt hatten. Einzelne Fälle sind bei Reisenden vorgekommen, der letzte größere Ausbruch ereignete sich in Angola im Jahr 2004 und markierte die Ausbreitung des Virus aus Zentralafrika in den Westen. Fledermäuse gelten als Reservoir und können das Virus über größere Strecken transportieren.

Zum Zeitpunkt, da dies geschrieben wird, belastet einer der größten Ebola-Ausbrüche zum ersten Mal Westafrika, besonders die Länder Guinea, Sierra Leone und Liberia, also Regionen, die bisher schon mit Lassa-Virus-Erkrankungen zu kämpfen hatten. In einer der Erstbeschreibungen des Ebola-Virus wird es Maridi-Virus genannt, nach dem Ort des ersten wissenschaftlich bearbeiteten Ausbruchs im Sudan. Noch heute sind der Sudan, der Kongo und Uganda, also Zentralafrika, als das Kerngebiet für das Vorkommen anzusehen, zum Teil aufgrund der Assoziation der Infektion mit Menschenaffen, die ebenfalls erkranken und in einzelnen Ausbruchsregionen durch die Infektion stark reduziert wurden.

Als Hauptmechanismus der nicht nosokomialen Ausbrüche wird das Überspringen der Infektion von erlegten oder eingesammelten infizierten Menschenaffen („bushmeat") auf die Bevölkerung angesehen (• Abb. 21.10). Auch die weite Strecken zurücklegenden Fledermäuse dürften wie bei Marburg-Virus als Reservoirtiere und Vehikel für die Verbreitung dienen. Auch sie sind eine wichtige Eiweißquelle in der Ernährung vieler Menschen im tropischen Afrika.

In Ausbruchssituationen breitet sich die Erkrankung schnell von Mensch zu Mensch aus durch direkten Kontakt mit der Haut oder mit Körpersekreten, zum Beispiel im Rahmen von Beisetzungsritualen.

\section{- Klinik}

Unspezifische fieberhafte Allgemeinsymptome und Diarrhö stehen auch bei diesen Erkrankungen im Vorder- grund. Hinsichtlich einer möglichen Exposition ist wiederum die Anamnese wichtig. Temperaturen können subfebril sein. Unspezifische, flüchtige Exantheme sind beschrieben und können wie beim Dengue-Fieber auch makulopapulös sein (Nkoghe et al. 2012). Eine Pharyngitis kann quälend sein und die orale Flüssigkeitsaufnahme unmöglich machen.

Blutungen stehen nicht auffallend im Vordergrund. Sie können als Gingivablutungen, als wiederauftretende Blutung bei an sich schon abgeheilten Verletzungen oder post partum in Erscheinung treten (• Abb. 21.11). Eine aktivierte Gerinnung und Thrombozytopenie sind obligat, Leberbeteiligung ist die Regel.

\section{( Die Pathophysiologie der Erkrankung führt zu einem schnellen Multiorganversagen. Eine ZNS-Beteiligung äußert sich in Form von enzephalopathischen Stö- rungen oder Somnolenz.}

\section{- Differenzialdiagnose}

Differenzialdiagnostisch kommen die bei Dengue-Fieber genannten Erkrankungen infrage. Wiederum ist ein prompter Malariaausschluss erforderlich.

\section{- Diagnostik}

Die Reverse-Transkriptase-(RT-)PCR hat sich gerade im jüngsten Ausbruch als robuste, auch unter den Bedingungen im Busch anzuwendende Methodik erwiesen und kann mit einer Malaria-PCR kombiniert werden. Antigen- und Antikörpernachweis, Anzucht und Kultur sind möglich und in entsprechenden BSL-4-Laboratorien etabliert (in Deutschland in Marburg und Hamburg).

\section{- Therapie}

Sie findet bei beiden Infektionen außerhalb der Endemiegebiete ausschließlich in dafür vorgesehenen Behandlungszentren unter Unterdruckbedingungen statt. Strikte Iso- 

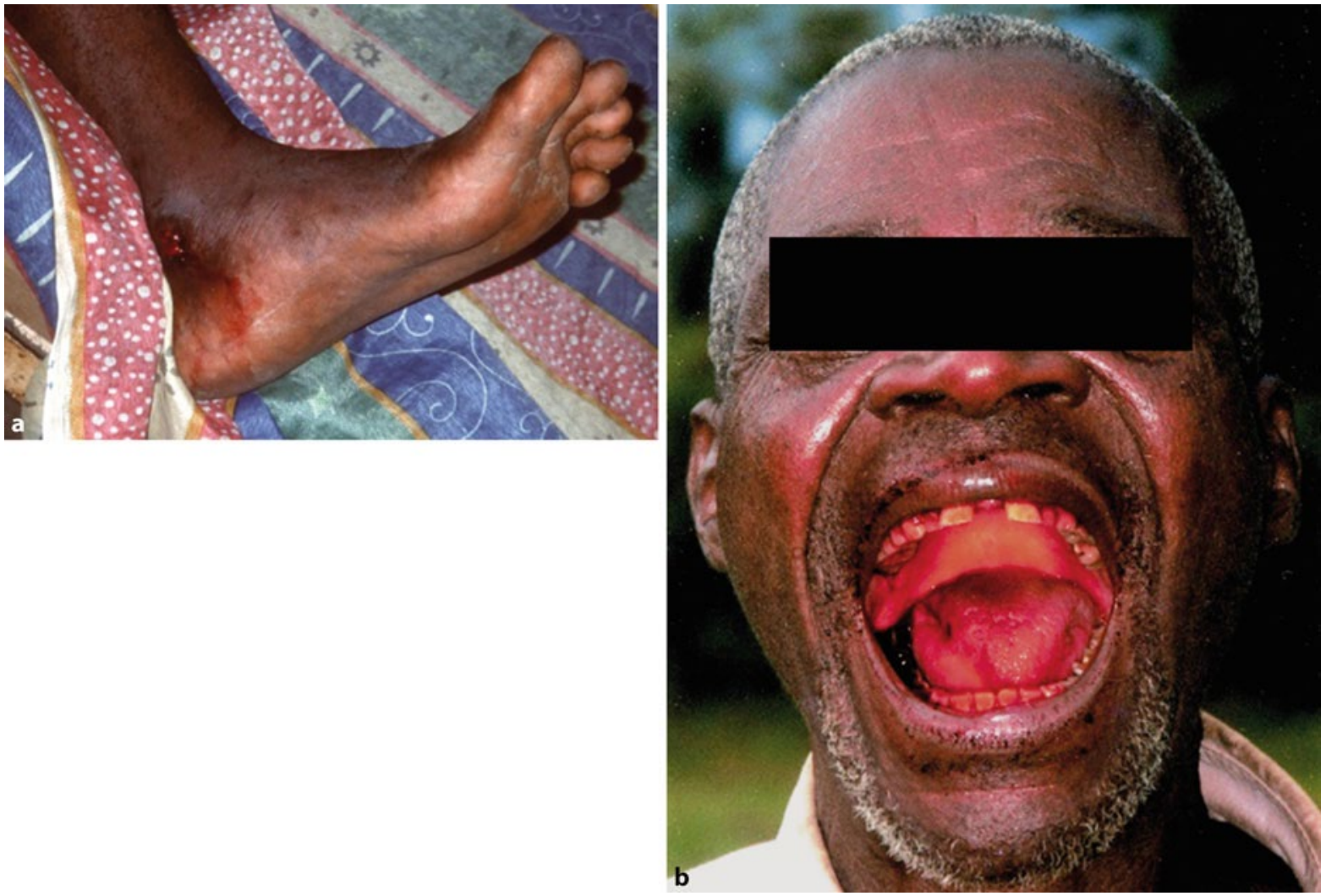

- Abb. 21.11a,b Blutungen bei akuter Ebola-Infektion. a Rezidivblutung einer alten Verletzung, b Gingivablutung. (@ Hinrich Sudeck, Hamburg)

lierungsmaßnahmen dienen nicht nur dem Selbstschutz, sondern auch der Unterbrechung der Infektionskette.

Bisher hat sich keine Therapie etabliert, allerdings sind eine Reihe von experimentellen Vakzinen für Ebola- und Marburg-Virus, teils vektorbasiert oder auf RNA-Basis, beschrieben. Im Tierversuch erwies sich die Gabe von monoklonalen Antikörpern als erfolgreich (Saphire 2013). Ein Nematodenprotein, das zur Gerinnungshemmung klinisch bei anderen Indikationen verwendet wird, und weitere antiviral wirksame Substanzen wurden bisher bei Menschen noch nicht eingesetzt. Der große Ausbruch in Westafrika hat zum Beginn unterschiedlicher Studien beigetragen, erste Ergebnisse sollten im Verlauf des Jahres 2015 vorliegen.

Die Letalität liegt je nach Virustyp und Qualität der supportiven Behandlung bei 25-90\%. Innere Blutungen kommen vor, sind aber nicht offensichtlich, und Verbluten ist nicht notwendigerweise die Todesursache. Der Tod tritt etwa 6-10 Tage nach Symptombeginn in Form eines Multiorganversagens ein, ähnlich wie bei einer septischen Erkrankung.

\subsection{Andere Erreger von viralen hämorrhagischen Fiebern}

\subsubsection{Gelbfieber}

Gelbfieber wird von einem Flavivirus hervorgerufen, es kommt zwischen dem 10. südlichen und dem 15. nördlichen Breitengrad ab Panama und in Südamerika sowie in der Subsahara vor. Es wird von Mücken der Gattungen Aedes (Stegomyia und Haemagogus) übertragen. Reservoire sind der Mensch und Affen, daher ist die Übertragung sowohl in ländlichen als auch in (groß-)städtischen Regionen möglich. Importe sind aufgrund der effektiven, weltweit zur Verfügung stehenden Lebendvakzine extrem selten. Typisch für das klinische Bild sind Ikterus, Nierenversagen und Blutungsneigung. Wichtige Differenzialdiagnosen sind alle in den vorherigen Abschnitten genannten Erkrankungen. Die Diagnostik muss unter BSL-3-Bedingungen erfolgen, die Therapie erfolgt rein symptomatisch in spezialisierten Zentren. Die Letalität der häufig zweiphasig verlaufenden Erkrankung liegt bei 10-50\%. 


\subsubsection{Lassa-Fieber}

Es wird durch ein Arenavirus verursacht, das in Westafrika endemisch ist und ein Reservoir in Nagern hat. Die Infektion führt nach unspezifischem Beginn zu Pharyngitis, Konjunktivitis und Multiorganversagen mit ausgeprägter Leberbeteiligung und möglichen Blutungen. Importe kamen in den letzten Jahren vereinzelt auch in Deutschland vor. Die Diagnostik erfolgt analog wie bei den anderen Viren, zur Therapie und auch zur Postexpositionsprophylaxe wird Ribavirin eingesetzt.

\subsubsection{Südamerikanische hämorrhagische Fieber}

Das argentinische, das bolivianische und das venezolanische hämorrhagische Fieber werden von Arenaviren unterschiedlicher Spezies verursacht. Diese Erkrankungen kommen in umschriebenen ländlichen Regionen der jeweiligen Länder vor und sind daher überwiegend eine Gefahr für die bäuerliche Bevölkerung. Hautsymptome in Form von Rötung und Petechien sowie Schleimhautbeteiligung mit Vesikeln, Enanthem und Konjunktivitis sollen häufig sein. Bei Verdachtsfällen sollte eine prompte Kontaktaufnahme mit einem Tropeninstitut erfolgen. Die Letalität liegt bei $10-20 \%$.

\section{Literatur}

Literatur zu Abschn. 21.2

Huy NT, Van Giang T, Thuy DH, Kikuchi M, Hien TT, Zamora J, Hirayama K (2013) Factors associated with dengue shock syndrome: a systematic review and meta-analysis. PLosNegl Trop Dis 7(9):e2412

Machado CR, Machado ES, Rohloff RD, Azevedo M, Campos DP, de Oliveira RB, Brasil P (2013) Is pregnancy associated with severe dengue? A review of data from the Rio de Janeiro surveillance information system. PLosNegl Trop Dis 7(5):e2217

WHO (2014) Dengue, countries or areas at risk, 2013. http://gamapserver.who.int/mapLibrary/Files/Maps/Global_DengueTransmission_ITHRiskMap.png?ua=1

Zang F, Kramer CV (2014) Corticosteroids for dengue infection. Cochrane Database Syst Rev 7:CD 003488 (epub ahead of print)

Literatur zu Abschn. 21.3

Bond A (2012) Man, 38, dies from deadly tropical disease after returning to the UK from Afghanistan. Mail online 6 Oct. 2012. www.dailymail. co.uk/news/article-2213758/Crimean-Congo-haemorrhagic-fever-Man-dies-tropical-disease-returning-UK-Afghanistan.html

Dokuzoguz B, Celikbas AK, Gök ŞE, Baykam N, Eroglu MN, Ergönül Ö (2013) Severity scoring index for Crimean-Congo hemorrhagic fever and the impact of ribavirin and corticosteroids on fatality. Clin Infect Dis 57(9):1270-1274

Duygu F, Kaya T, Baysan P (2012) Re-evaluation of 400 Crimean-Congo hemorrhagic fever cases in an endemic area: Is Ribavirin treatment suitable? Vector-borne and zoonotic Diseases 12(9):812-816
Elata AT, Karsany MS, Elageb RM, Hussain MA, Eltom KH, Elbashir MI Aradaib IE (2011) A nosocomial transmission of crimean-congo hemorrhagic fever to an attending physician in North Kordufan, Sudan. Virol J 8:303

Estrada-Peña A, Palomar AM, Santibáñez P, Sánchez N, Habela MA Portillo A, Romero L, Oteo JA (2012) Crimean-Congo hemorrhagic fever virus in ticks, Southwestern Europe 2010. Emerg Infect Dis 18(1):179-180

WHO (2008) Geographic Distribution of Crimean-Congo Haemorrhagic Fever. http://www.who.int/csr/disease/crimean_congoHF/Global_ CCHFRisk_20080918.png?ua=1

Literatur zu Abschn. 21.4

Nkoghe D, Leroy EM, Toung-Mve M, Gonzalez JP (2012) Cutaneous manifestations of filovirus infections. Int J Dermatol 51(9):1037-1043

Weiterführende Literatur

Burchard GD, Günther S, Schmidt-Chanasit J (2010) Virale hämorrhagische Fieber. In: Burchard GD, Löscher T (Hrsg) Tropenmedizin in Klinik und Praxis. Thieme, Stuttgart

Clark DV, Jahrling P, Lawler V (2012) Clinical Management of Filovirus-Infected Patients. Viruses 4:1668-1686

Hoenen T, Groseth A, Feldmann A (2012) Current Ebola vaccines. Expert Opin Biol Ther 12(7):859-872

Mehedi M, Groseth A, Feldmann H, Ebihara H (2011) Future clinical aspects of Marburg hemorrhagic fever. Virol 6(9):1091-1106

Paessler S, Walker DH (2013) Pathogenesis of the viral hemorrhagic fevers. Annu Rev Pathol 8:411-440

Saphire EO (2013) An update on the use of antibodies against the filoviruses. Immunotherapy 5(11):1221-1233

WHO (2009) Dengue Guidelines for Diagnosis, Treatment, Prevention and Control. WHO, Geneva 